\title{
THE USE OF THE POSITIVITY METHOD TO APPROXIMATE THE COMPLETE O.D.F.
}

\author{
F. WAGNER \\ CMS/GM2P, Université de Metz, Ile du Saulcy, \\ 57045 Metz-cedex, France
}

(Received 30 January 1996)

\begin{abstract}
The mathematical problems, in the field of texture analysis, which remain still unsolved are briefly discussed. The importance of the positivity condition and its use in the harmonic method are then explained.
\end{abstract}

KEY WORDS: Texture analysis, ODF-calculation, positivity technique

\section{INTRODUCTION}

This paper will give first a brief survey of some mathematical problems of quantitative texture analysis which still remain open. It will then explain the state of the art in the 'classical' harmonic method with a special attention regarding the question of positivity.

There are two main ways of collecting experimental data for texture analysis. The first one consists in measuring individual orientations (by using the E.B.S.D. in a scanning electron microscope for example). The second one consists in measuring pole figures by using neutron or X-ray diffraction. In both cases there are some mathematical problems when an O.D.F., $\mathrm{f}(\mathrm{g})$, has to be calculated from such experimental data.

\section{Texture Analysis by Using Individual Orientation Measurements}

It is assumed here that the result of an experiment consits in a set of $\mathrm{N}$ measurements $\left\{g_{i}, w_{i} ; 1 \leq i \leq N\right\}$ with $g_{i}$ the orientation of a grain and $w_{i}$ the relative volume of this grain. Most of the time, the number of measurements, $\mathrm{N}$, is limited because they require that an operator selects the grains on which a Kikuchi pattern is formed which is time consuming. Typical values for $\mathrm{N}$ in the litterature are in the range 500 to 1000 . The development of automatized EBSD systems will of course allow to increase this number in routine operations (see Adams 1994, Kriegerlassen 1995).

The O.D.F., $\mathrm{f}(\mathrm{g})$, is usually calculated by summing $\mathrm{N}$ bell-shaped model functions centered at $\mathrm{g}_{\mathrm{i}:}$

$$
f(g)=\sum_{i=1}^{i=N} w_{i} f^{M}\left(g_{i}, b_{i}, g\right)
$$

One must then choose a type of model function (Gauss-type or Lorentz-type for example) as well as a parameter $b_{i}$ describing the spread around $g_{i}$ (in a Gauss-type model function 
$b_{i}$ is the full half width). Because such model functions are always positive, the O.D.F., as defined by equation (1), is also positive. The most difficult questions are:

- how many measurements, N, must be carried out in order to reach a given resolution power in the O.D.F.?

- and accordingly which $b_{i}$ values have to be used for this resolution power?

Obviously the answer to the first question depends on the texture itself. This can be easily understood by considering the two following extreme cases. If all the grains have the same orientation $\mathrm{g}_{0}$, one measurement is sufficient to construct the correct O.D.F. which is a Dirac function $\delta\left(\mathrm{g}_{0}\right)$. On the opposite hand, if the texture is a random one, one needs at least $\mathrm{N}_{0}$ measurements; $\mathrm{N}_{0}$ is obtained by dividing the volume of the whole orientation space by the wished resolving power.

From such considerations a relationship between the number of measurements $\mathrm{N}$ and $b_{i}$, the spread parameter of the model functions, has been proposed by Wagner et al. 1981. This relationship involves an empirical parameter characterizing the sharpness of the texture. More recently Pospiech et al. (1994) have proposed to follow the evolution of a kind of sharpness parameter with increasing $\mathrm{N}$ values; when this parameter becomes nearly constant, the value of $\mathrm{N}$ is considered as sufficient to construct the O.D.F. according to formula (1). It should be pointed out that these works do not solve the problem completely. Mathematical considerations upon such statistic problems should lead very likely to progresses in the determination of O.D.F.'s from individual measurements.

\section{Texture Analysis from Pole Figures Data}

The data which are usually collected for texture analysis by using neutron or X-ray diffraction and a four circles goniometer consist in a set of incomplete pole figures $\mathrm{I}_{\mathrm{hi}}(\mathrm{y})$ where hi denotes a given $\{\mathrm{hkl}\}$ plane family and y a given direction in the specimen coordinate system. These pole figures give after normalization the experimental pole densities $\mathrm{P}_{\mathrm{hi}}(\mathrm{y})$ :

$$
P_{h i}(y)=N i I_{h i}(y)
$$

Many ways have been proposed to calculate the normalization factors $\mathrm{Ni}$ (Humbert and Bergmann, 1980; Van Houtte 1980; Liang et al., 1981; Dahms and Bunge, 1986; Wagner and Humbert, 1987). There is an integral relation between the pole density and the searched O.D.F., $\mathrm{f}(\mathrm{g})$. It is now well established that this integral equation does not lead to an unique solution because of the existence of a symmetry center either due to the crystal structure of introduced by the diffraction (Matthies, 1979; Esling et al., 1980; Bunge et al., 1980; Esling, 1981). This ambiguity can be presented as follows. The O.D.F., $\mathrm{f}(\mathrm{g})$, can be split into two parts, the directly determinable part, $\tilde{\mathrm{f}}(\mathrm{g})$, and the not directly determinable part, $\widetilde{\mathrm{f}}(\mathrm{g})$ :

$$
f(g)=\tilde{f}(g)+\tilde{\tilde{f}}(g)
$$

They are often referred as the even and odd parts because their expansions on generalized spherical harmonic functions $T_{1}^{\mathrm{mn}}(\mathrm{g})$ require functions with even and odd 1 integers respectively. They obey the following integral equations: 
and

$$
\oint_{\text {hi } / / y} \tilde{f}(g) d g=P_{h i}(y)
$$

$$
\oint_{\text {hi } / / y} \tilde{\text { f }} \mathrm{dg}=0
$$

The consequence is that there are several functions $\mathrm{f}(\mathrm{g})$, in fact a field of functions, with the same even part i.e. several functions which correspond to a given set of pole figures (see examples in Matthies and Wagner, 1981). There are therefore two questions in this problem of determining an O.D.F. from a given set of pole figures:

- how to select one solution among all the possible ones?

- what is the variation width of the field of solutions?

No satisfying answer has be given up to now to the second question although some attempt has been made in this way (Schaeben, 1994). It is only known qualitatively from calculations with model functions that the width of variation of the solution field is very narrow for a sharp texture and somewhat larger for a smooth texture. A mathematical procedure allowing to give the answer in the form $f(g) \pm \Delta f(g)$ is still to be discovered.

Many ways have been found to answer the first question i.e. to determine one O.D.F. from a set of measured pole figures. In all the cases they are based, on one hand, on the fact that the searched function, $f(g)$, must be greater or equal zero (according to the definition of the O.D.F.) and, on the other hand, on a particular condition which can be explicit or hidden in the determination procedure. In the framework of the socalled discrete methods (both the O.D.F. and the pole figures are considered as staircase functions) the following conditions have been used: maximal phon and a minimum number of sharp peaks (Wimv method by Matthies 1988); maximum entropy (Schaeben, 1990); lowest texture index (hidden condition in the vector method, Vadon 1981). In the framework of the harmonic method several possibilities have also been evidenced: assumption that the O.D.F. is identical to the square of a function (Van Houtte, 1983), or to an exponential form (Van Houtte, 1991); assumption that the O.D.F. is a set of Gaussian peaks whose parameters must be optimized in each case (Lücke et al., 1981). We will give hereafter some more details about an other way of using positivity conditions in the harmonic method and will referred to this method as the "classical' harmonic method.

\section{THE 'CLASSICAL' HARMONIC METHOD}

The calculation of the O.D.F. is separated into two stages. First the even part, $\tilde{\mathrm{f}}(\mathrm{g})$, is determined by solving the equation (4). Then an odd part, $\widetilde{\tilde{f}}(\mathrm{~g})$, is calculated in order to obtain the O.D.F., $\mathrm{f}(\mathrm{g})$.

\section{Calculation of the even part, $\tilde{f}(g)$}

The integral equation (4) can be solved by using for both functions, $\tilde{\mathrm{f}}(\mathrm{g})$ and $\mathrm{P}_{\mathrm{hi}}(\mathrm{y})$, series expansions on the bases of generalized spherical harmonics and spherical harmonics respectively (Bunge, 1965; Bunge, 1969). This is done in fact with a least square scheme. As soon as a solution, $\tilde{\mathrm{f}}(\mathrm{g})$, has been calculated from the experimental 
data, it is possible to recalculate any direct or inverse pole figure. The mean difference between the experimental pole densities and the recalculated ones is the so called error coefficient, RP(hi), for each pole figure.

Because of the errors in the experimental data, the pole figures which are recalculated from $\tilde{\mathrm{f}}(\mathrm{g})$ can exhibit negative values in some areas. Dahms and Bunge, 1988, have proposed to use a positivity criterium in an iterative scheme in order to improve the solution regarding this drawback of negative values in the recalculated pole figures. This 'first floor positivity criterium' works in the following way: when a solution, $\widetilde{\mathrm{f}}(\mathrm{g})$, has been found, pole figures are recalculated (the ones which have been used as input data and eventually some supplementary ones). A set of complete pole figures is then constituted for a new analysis; for the measured incomplete pole figures, the inner part consists in the normalized measured data whereas the outer part is formed with the recalculated values. When negative values exist in the recalculated values they are replaced by zero. In a similar way the supplementary pole figures are recalculated ones in which negative values are replaced by zero. This set of complete pole figures is then used as input data for the calculation of a new solution $\tilde{\mathrm{f}}(\mathrm{g})$. This iterative procedure is stopped when no more negative values exist in the recalculated pole figures or when no improvement is obtained in decreasing these negative values.

Checking the validity and the interest of such an iterative procedure is somewhat complicated. It requires indeed to consider data which contain some errors and to know the true solution, $\mathrm{f}(\mathrm{g})$. Mellab, 1987, has performed such tests: by considering a model O.D.F., he has calculated the even part, $\tilde{\mathrm{f}}(\mathrm{g})$, and the corresponding pole figures. These pole figures were then deliberately falsified and used as input 'experimental' data so that it was possible to follow how the iterative procedure improves the calculated solution for the even part of the O.D.F. In such a case both apparent error coefficients (regarding the falsified data), $\mathrm{RP}^{\mathrm{a}}$, and the true error coefficients (regarding the true data), $\mathrm{RP}^{\text {true }}$, can be calculated.

Figure 1 illustrates schematically the result of such a calculation. $\mathrm{P}_{\text {hi }}^{\text {true }}$ holds for the true pole figures, $\mathrm{P}_{\mathrm{hi}}^{\text {exp }}$ for the falsified ones, $\mathrm{P}_{\mathrm{hi}}^{\text {rec }}\left(\tilde{\mathrm{f}}_{\mathrm{g}}(\mathrm{g})\right)$ for the recalculated ones after analysis without the positivity criterium and $P_{h i}^{\text {rec }}\left(\tilde{f}_{n}(g)\right)$ for the recalculated ones after analysis with the positivity criterium. Whereas the apparent error coefficients, RPa, are not significantly changed, the true error coefficients, $\mathrm{RP}^{\text {true }}$, are improved by using this 'first floor positivity criterium'.

Nevertheless this iterative scheme with the 'first floor positivity criterium' does not always converge to a solution which corresponds to recalculated pole figures without any negative value. The convergency seems to be influenced by the quality of the experimental data. Large errors in the experimental data are a 'brake' for the convergency. This general question of the sensitivity of a texture analysis method to the errors in the data remains widely unexplored.

\section{Calculation of the O.D.F., $f(g)$}

Once the even part, $\tilde{\mathrm{f}}(\mathrm{g})$, has been calculated, the O.D.F. has to be determined. In the 'classical harmonic method' this means that an odd part, $\widetilde{\mathrm{f}}(\mathrm{g})$, must be added to the even one. This is performed on the basis of a positivity criterium (Dahms and Bunge, 1988; Wagner and Dahms, 1991) which will be called here 'second floor positivity criterium'. The O.D.F., because of its definition, must indeed be greater or equal to zero. The 'second floor positivity criterium' is also worked out in an iterative way. The iterative scheme is as follows: 


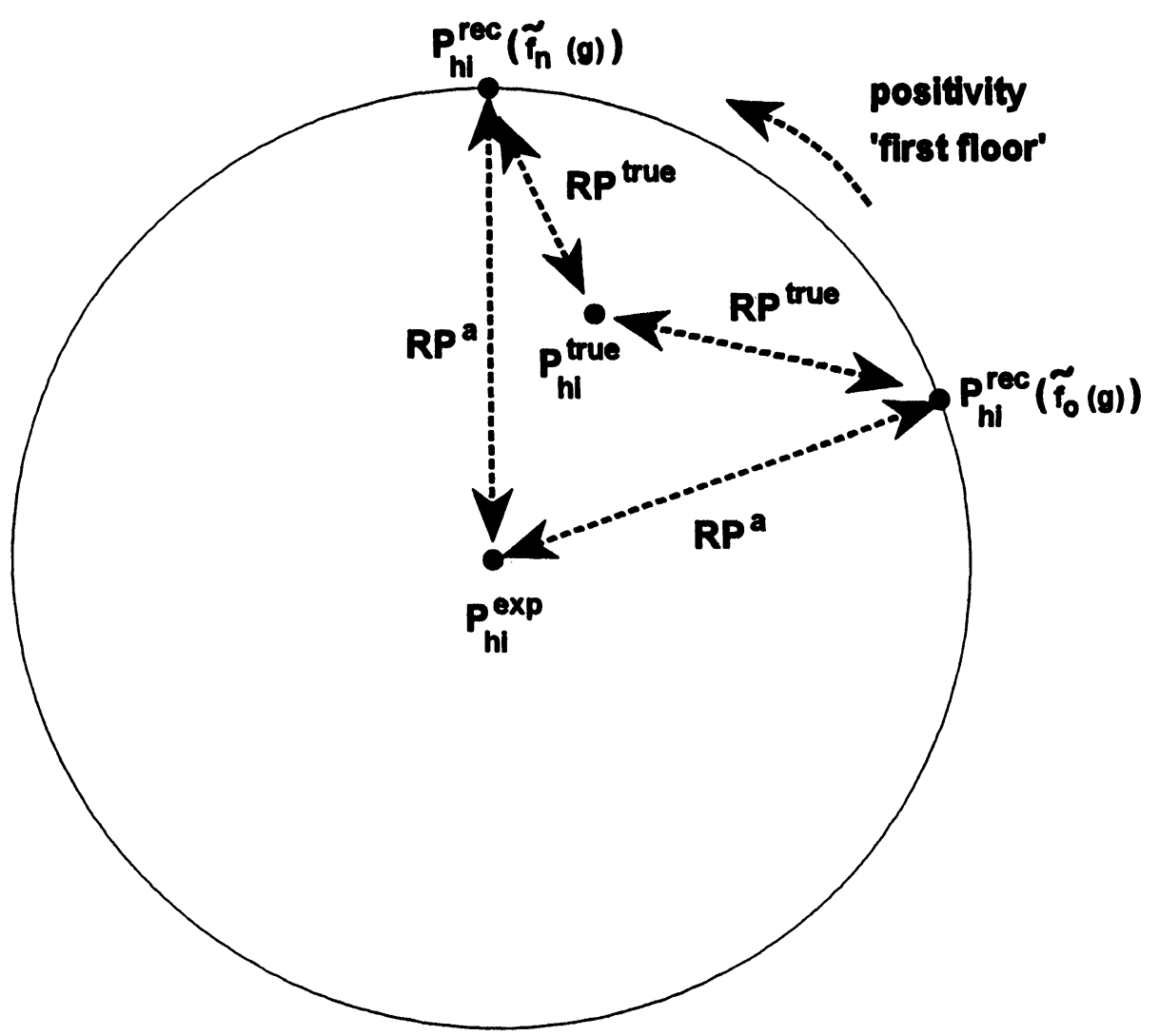

Figure 1 Schematic representation of the effect of the 'first floor positivity criterium'.

with

$$
\mathrm{f}_{\mathrm{n}}(\mathrm{g})=\mathrm{f}_{\mathrm{n}-1}(\mathrm{~g})+\lambda_{\mathrm{n} \tilde{\mathrm{f}} \mathrm{n}}(\mathrm{g})
$$

and

$$
\mathrm{f}_{0}(\mathrm{~g})=\tilde{\mathrm{f}}(\mathrm{g})
$$

$$
\begin{array}{ll}
\hat{\mathrm{f}}_{\mathrm{n}}(\mathrm{g})=\mathrm{f}_{\mathrm{n}-1}(\mathrm{~g}) & \text { if } \mathrm{f}_{\mathrm{n}-1}(\mathrm{~g}) \geq 0 \\
\hat{\mathrm{f}}_{\mathrm{n}}(\mathrm{g})=0 & \text { if } \mathrm{f}_{\mathrm{n}-1}(\mathrm{~g})<0
\end{array}
$$

In equation (6), the parameter $\lambda_{\mathrm{n}}$ is used to increase the convergency speed (Wagner et al. 1991). There is in fact a hidden condition in this procedure. Because it consists in minimizing the domain with negative values, the selected O.D.F., among all the possible ones of the field of solutions, is the one which is the closest to the even part, $\tilde{\mathrm{f}}(\mathrm{g})$ (Wagner et al. 1991).

The efficiency of this method has been demonstrated by using model O.D.F.'s (Dahms and Bunge 1988, Wagner and Dahms, 1991). When real data are used, it is not always possible to build an O.D.F. which is fully positive. This is linked once more with the 
quality of the experimental data. The starting point of this iterative positivity technique is indeed the even part, $\widetilde{\mathrm{f}}(\mathrm{g})$, which is calculated from the data as explained in the previous section. The more precise the even part, $\tilde{\mathrm{f}}(\mathrm{g})$, the more efficient the iterative positivity technique. This is illustrated at Figure 2. It concerns an annealed low carbon steel for which three incomplete pole figures have been measured. Figure 2a) shows the O.D.F. obtained with the 'second floor positivity criterium' when starting from an even part. $\tilde{\mathrm{f}}(\mathrm{g})$, calculated without the 'first floor positivity criterium'. In this case the extremal values are $f_{\max }(g)=9.7$ and $f_{\min }(g)=-1.3$. Figure $\left.2 b\right)$ shows the O.D.F. obtained with the 'second floor positivity criterium' when starting from an even part, $\tilde{\mathrm{f}}(\mathrm{g})$, calculated with the 'first floor positivity criterium'. In this second case the extremal values are $f_{\max }(g)=8.6$ and $f_{\min }(g)=-0.1$. It is clear that the second calculation, in which both positivity criteria are activated, leads to a more realistic result in that sense that the obtained O.D.F. is nearly positive or null in the whole orientation space.

Once more, a precise correlation between the quality of the experimental data and the convergency in the iterative positivity technique remains to be established.

\section{CONCLUSION}

Although a lot of papers has been devoted to quantitative texture analysis, several mathematical problems remain unsolved in this field. When the experimental data consist in a set of orientations, the main question in calculating the corresponding O.D.F. is the relationship between the number of the available measurements and the resolving power in the orientation space. As explained in the first section, this resolving power

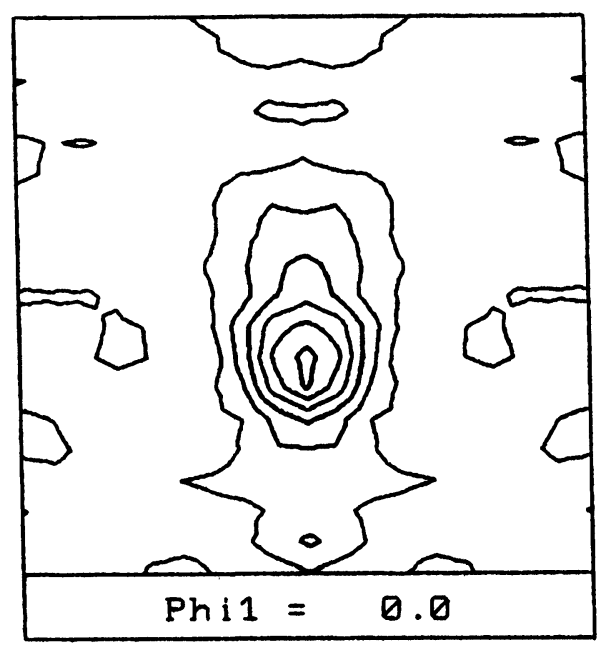

a)

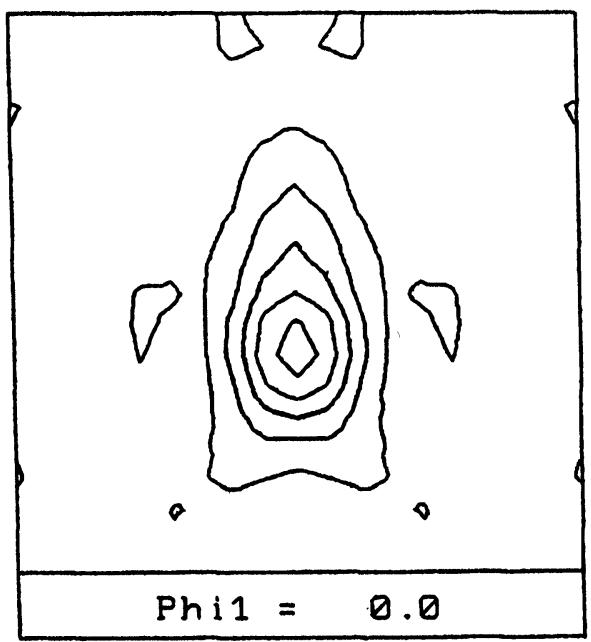

b)

Figure 2 The section $\varphi 1=0^{\circ}$ of an O.D.F. of a low carbon steel calculated a) without a 'first floor positivity criterium'; b) with a 'first floor positivity criterium'. 
depends on the searched unknown O.D.F. itself for a given number of measurements, which increases the complexity of the problem. When the experimental data consists in a set of pole figures there is a field of O.D.F.'s which explain these pole figures. No satisfying way has been found up to now to calculate its 'width' which here also depends on the texture itself. The goal is restricted at the time to the calculation of one solution in this field. Many methods have been proposed for such a purpose. All of them take into consideration the positivity of the O.D.F. according to its definition. The advantages of the harmonic method are well known. It does not assume any particular condition for the solution because the functions which are used for the series expansions are bases. This solution consists thus in a set of coefficients which can be correlated with anisotropic properties of polycrystals. The 'classical' harmonic method uses positivity criteria at two stages: one when calculating the even part which explains the pole figures and one when building the 'complete' solution i.e. the O.D.F. Both are necessary to obtain a satisfactory solution as it was demonstrated in the previous sections.

One problem remains unsolved in the harmonic method as well as in the other methods of texture analysis: how the errors and the resulting incompatibilities in the experimental pole figures influence the calculated O.D.F. This should be also an investigation field in the future.

\section{References}

Adams, B. (1994). Proceedings of ICOTOM 10, Trans Tech Publications, p. 31.

Bunge, H. J. (1965). Zeit. Metallkde, 56, p. 872

Bunge, H. J. (1969). «Mathematische Methoden der Texturanalyse». Akademie-Verlag Berlin.

Bunge, H. J., Esling, C. and Muller, J. (1981). J. Acta Cryst., A37, 889.

Dahms, M. and Bunge, H. J. (1986). Textures and Microstructures, 6, p. 167.

Dahms, M. and Bunge, H. J. (1988). Textures and Microstructures.

Esling, C., Bunge, H. J. and Muller, J. (1980). J. Phys. Let., 41, p.L-543.

Esling, C. (1981). thèse de doctorat d'état, Université de Metz (France).

Humbert M. and Bergmann, H. W. (1980). J. Appl. Cryst., 13, p. 500.

Krieger Lassen, N. C. (1995). Proceedings of the 16th Risö International Symposium, p. 405.

Liang, Z., Xu, J. and Wang F. (1981). Proceedings of ICOTOM 6, The Iron and Steel Institute of Japan, II, p. 1259.

Lücke, K., Pospiech, J., Virnich, K. H. and Jura, J. (1981), Acta Met., 29, p. 167.

Matthies, S. (1979). Phys. stat. sol. (b), 92, p. k135.

Matthies, S. and Wagner, F. (1981). Phys. stat. sol. (b), 107, p. 591.

Matthies, S. (1988). Proceedings of ICOTOM 8, ed. J. S. Kallend and G. Gottstein, p. 38.

Mellab, F. (1992). Phd thesis, Université de Metz (France).

Pospiech J., Jura J. and Gottstein G. (1994). Proceedings of ICOTOM 10, Trans Tech Publications, p. 407.

Schaeben, H. (1988). Phys. stat. sol. (b), 148, p. 63.

Schaeben, H. (1992). Textures and Microstructures, 21, p. 55.

Vadon, A. (1981). thèse de doctorat d'état, Université de Metz (France).

Van Houtte, P. (1980). Mater. Sci. Eng., 43, p. 7.

Van Houtte, P. (1983). Textures and Microstructures, 6, p. 1.

Van Houtte, P. (1991). Textures and Microstructures.

Wagner, F., Wenk. H. R., Esling, C. and Bunge H. J. (1981). Phys. stat. sol. (a), 67, p. 269.

Wagner, F. and Humbert, M. (1987). Textures and Microstructures, 7, p. 115.

Wagner, F. and Dahms, M. (1991). in "Advances and applications of quantitative texture analysis», ed. H. J. Bunge and C. Esling, DGM Oberursel, p. 101.

Wagner, F., Humbert M., Muller, J. and Esling, C. (1991). Europhys. Lett., 11, p. 479. 